\author{
Marquette University \\ e-Publications@Marquette
}

Electrical and Computer Engineering Faculty Research and Publications

Electrical and Computer Engineering,

Department of

\title{
Induction Machine Broken-bar Fault Diagnosis Using the Rotor Magnetic Field Space-vector Orientation
}

\author{
Behrooz Mirafzal \\ Marquette University \\ Nabeel Demerdash \\ Marquette University, nabeel.demerdash@marquette.edu
}

Follow this and additional works at: https://epublications.marquette.edu/electric_fac

Part of the Computer Engineering Commons, and the Electrical and Computer Engineering Commons

\section{Recommended Citation}

Mirafzal, Behrooz and Demerdash, Nabeel, "Induction Machine Broken-bar Fault Diagnosis Using the Rotor Magnetic Field Space-vector Orientation" (2004). Electrical and Computer Engineering Faculty Research and Publications. 193.

https://epublications.marquette.edu/electric_fac/193 
Marquette University

e-Publications@Marquette

\section{Department of Electrical and Computer Engineering Faculty Research and Publications/College of Engineering}

This paper is NOT THE PUBLISHED VERSION.

Access the published version at the link in the citation below.

IEEE Transactions on Industry Applications, Vol. 40, No. 2 (2004, March-April): 534-542. DOI. This article is (C) Institute of Electrical and Electronic Engineers (IEEE) and permission has been granted for this version to appear in e-Publications@Marquette. Institute of Electrical and Electronic Engineers (IEEE) does not grant permission for this article to be further copied/distributed or hosted elsewhere without the express permission from Institute of Electrical and Electronic Engineers (IEEE).

\section{Induction Machine Broken-Bar Fault Diagnosis Using the Rotor Magnetic Field Space-Vector Orientation}

B. Mirafzal

Department of Electrical and Computer Engineering, Marquette University, Milwaukee, WI N.A.O. Demerdash

Department of Electrical and Computer Engineering, Marquette University, Milwaukee, WI

\section{Abstract:}

A new technique based on rotor magnetic field space vector orientation is presented to diagnose broken-bar faults in induction machines operating at steady state. In this technique, stator currents and voltages are used as inputs to compute and subsequently observe the rotor magnetic field 
orientation, which has a more significant "swing-like" pendulous oscillation in case of broken-bar faults than in healthy operation. It will be shown here that the range of this "pendulous oscillation" is a function of the number of broken bars. Also in this technique, it was found that an inter-turn shorted stator-winding fault, which exhibits similar pendulous oscillation, could be distinguished from rotor broken-bar faults through the use of a variance index. In order to validate this method, experimental evidence is given here for several broken-bar cases in a 2-hp three-phase two-pole squirrel-cage induction machine.

\section{SECTION I Introduction}

IN THE PAST two decades, there have been many investigations on fault diagnostics in electric machines, especially squirrel-cage induction motors [1]-[2][3][4][5][6]. Because the squirrel-cage induction motor is presently one of the most widely used machines in industry, it is the most investigated machine with regard to motor fault diagnostics. A sudden motor failure may be very damaging or catastrophic in an industrial system, in which the electric motor is the prime mover. Consequently, these motor failures reduce productivity in industry. Hence, maintenance schedules are provided to proactively reduce or prevent these failures. Nevertheless, the probability of a sudden motor failure cannot be entirely ruled out. Moreover, increasing the frequency of scheduled maintenance increases the cost and decreases the productivity of a system. Accordingly, an online fault diagnostic system becomes a valuable tool to increase industrial efficiency and reliability. This paper propose a technique for fault diagnostic purposes which might help develop proactive "as needed" maintenance scheduling

Besides this introduction, the paper contains six additional sections. Section II theoretically shows that in case of broken-bar faults the rotor magnetic field oscillates around its original healthy case direction. Section IIIpresents the necessary formulations to obtain the rotor magnetic field orientation in a space-vector plain. In Section IV, the simulation results are analyzed and explained as to how interturn shorted stator-winding types of faults occurring simultaneously with broken-bar faults could be distinguished from pure broken-bar rotor faults. In Section V a 230-V 60-Hz two-pole 2-hp squirrel-cage three-phase induction motor was tested for faults constituting one through five rotor broken-bars. Also in this section, this new method's results of diagnosing the broken-bar faults for this 2-hp motor are compared with the results of applying the well-known fast Fourier transform (FFT) technique to the same set of motor phase currents under the same fault conditions, with its sideband frequency concepts. In Section VI a discussion of the merit of the present approach versus the FFT sideband approach is given

\section{SECTION II Rotor Magnetomotive Force (MMF) for a Case With a Broken -Bar Fault}

The objective of this section is to develop the idea of the "pendulous oscillation" of the rotor magnetic field in case of a broken-bar rotor fault. For demonstration purposes, consider the simple case of a three-phase two-pole squirrel-cage induction motor with eight rotor bars. This is a simplified example in order to easily grasp a good physical understanding of this "swing-like" pendulous oscillation phenomenon, and analytically verify this phenomenon and its angular swing magnitude as an index (measure) for the rotor broken-bar count and, hence, more specific fault diagnostics. In general, the 
fundamental components of the rotor loop currents in a healthy rotor case [Fig. 1(a)] can be expressed as follows:

$$
\begin{gathered}
i_{L k}(t)=I_{r} \cos \left(\omega_{r} t-\varphi-\frac{2 \pi(k-1)}{N_{b}}\right) \\
k=1,2, \ldots, N_{b}
\end{gathered}
$$

and accordingly, the rotor bar currents can be expressed in terms of the loop currents as follows:

$$
i_{b n}(t)=i_{L n-1}(t)-i_{L n}(t), n=1,2, \ldots, N_{b}
$$

where $\omega_{r}$ is the rotor angular frequency $(2 \pi s f), N_{b}$ is the number of rotor bars per pair of poles, while for this simplified example of Fig. $1, N_{b}=8$ bars. Here, $\varphi$ is a phase shift between the first rotor loop current and the reference. In this development, the reference is taken to be the stator voltage of phase $A$. In Fig. 1(b), it is assumed that the sixth bar is broken.

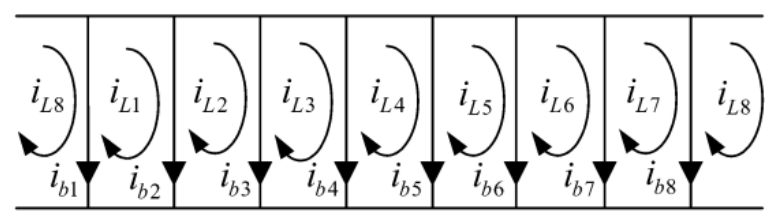

(a) Healthy Rotor Case

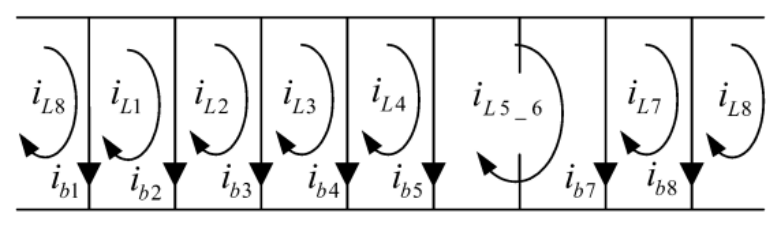

(b) One Broken-Bar Case

Fig. 1 Developed rotor cage circuits representation with a healthy and one broken bar for an eight-bar simplified example

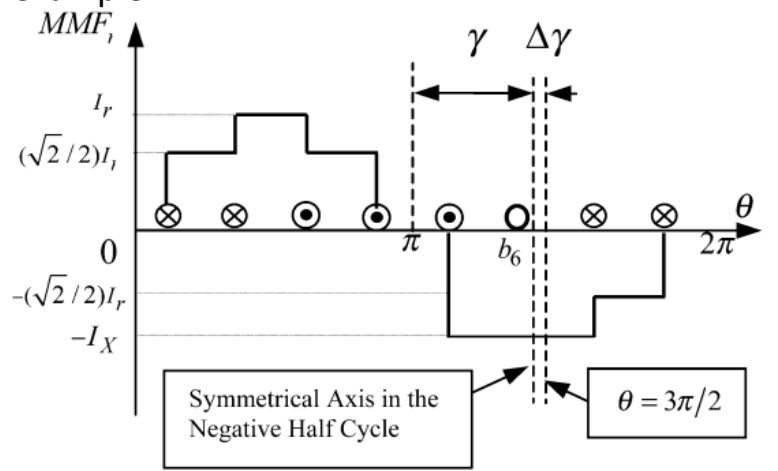

Fig. 2 Rotor MMF waveform of the eight-bar example at $t=0$, with one broken bar

Meanwhile, in this case, (1)only constitutes an approximation of the fundamental components of the healthy loop currents. However, this equation is not valid for the newly formed loop current resulting from the bar breakage. Specifically, this is the unhealthy loop current, $i_{L 5 \_}(t)$, see the developed 
schematic of the one broken-bar cage in Fig. 1(b). For purposes of this demonstration, let the aforementioned angle, $\varphi=3 \pi / 4 \mathrm{rad}$, and at time, $t=0$, the loop current, $i_{L 5_{-} 6}(0)=I_{X}$.

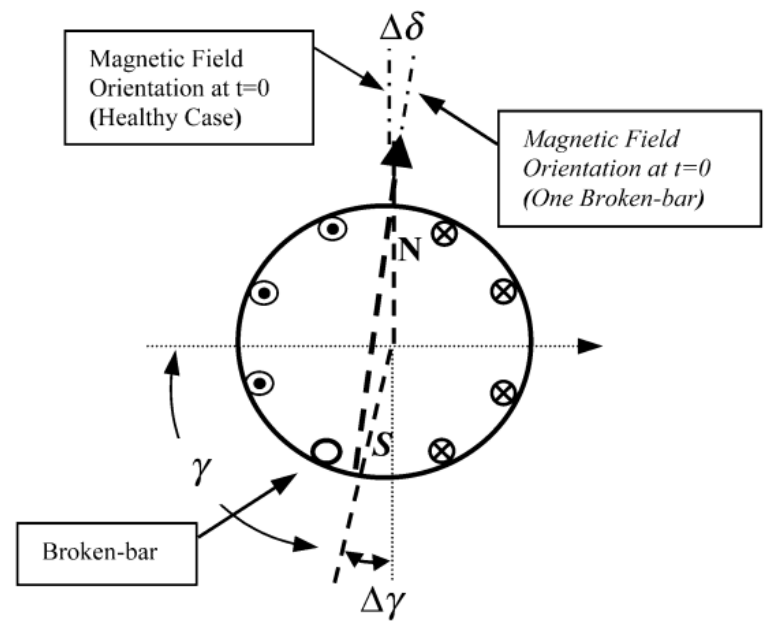

Fig. 3 Magnetic field orientation of the eight-bar example at $t=0$, with one broken bar

Accordingly, for the broken bar case of Fig. 1(b) the rotor MMF waveform at $t=0$ can be depicted as shown in Fig. 2 . The unknown value of the loop current, $i_{L 5_{-} 6}(0)=I_{X}$, can be calculated using the fundamental electromagnetic field concept, $\nabla \cdot \vec{B}=0$. This enables one to write the following relationship between the various loop currents of Fig. 1(b), assuming no axial flux exists in this case:

$$
\frac{\mu_{0} A_{\text {loop }}}{g}\left(i_{L 1}+i_{L 2}+i_{L 3}+i_{L 4}+2 i_{L 5_{-} 6}+i_{L 7}+i_{L 8}\right)=0
$$

where $A_{\text {loop }}$ is the rotor loop area, and $g$ is the air-gap height. Solving $(3)$ at $t=0$, using substitutions for $i_{L 1}$ through $i_{L 4}, i_{L 7}$, and $i_{L 8}$ from $(1)$, in conjunction with the necessary algebraic manipulations gives

$$
I_{X}=\left(\frac{1}{2}+\frac{\sqrt{2}}{4}\right) I_{r}
$$

It should be emphasized here that the loop currents contain a set of higher order harmonics, where in these calculations only their fundamental components are considered. Moreover, the currents in the healthy loops will be affected slightly in the presence of the broken bar, where those effects are neglected in these calculations for only qualitative conceptualization purposes of the rotor field's pendulous oscillation phenomenon. That is, these assumptions qualitatively demonstrate the rotor magnetic field pendulous oscillations in the presence of broken-bar faults

Now, from (1) (2), and (4), the rotor MMF profile at $t=0$ can be depicted as shown in Fig. 2. As one can see from Fig. 2, the location of the magnetic axis (or center of mass) of the negative half cycle of 
the rotor MMF is shifted to the left side of the original axis by a nonzero angular value, $\Delta \gamma$. In order to calculate this angular shifted, $\Delta \gamma$, see Fig. 2, the angle $\gamma$, which defines the location of the "center of mass" of the distorted MMF, can be determined as follows:

$$
\left(\gamma-\frac{\pi}{8}\right) I_{X}=\left(\frac{5 \pi}{8}-\gamma\right) I_{X}+\left(\frac{2 \pi}{8}\right)\left(\frac{\sqrt{2}}{2}\right) I_{r} .
$$

Upon substitution for $I_{X}$ from its above-determined value in (4), the angle, $\gamma$, locating the magnetic axis will have a value as follows: $\gamma=(0.957)(\pi / 2)$. Subsequently, the resulting angular shift, $\Delta \gamma$, of the magnetic axis for this half waveform caused by the sixth bar breakage can be obtained as follows; $\Delta \gamma=(\pi / 2)-(0.957)(\pi / 2)=(0.043)(\pi / 2)$. Fig. 3 shows the angular shift, $\Delta \gamma$, and the overall rotor magnetic field orientation (north pole to south pole line), which is now tending to the right side by an angle, $\Delta \delta=\Delta \gamma / 2$, at $t=0$. As time t progresses forward, $t>0$, the distortion in the profile of the rotor MMF will also be continuously modulated (changing) with time. This continuous change in the rotor MMF profile is equivalent to the rotor magnetic field displacement in subsequent leading and lagging manners around the original magnetic axis of the healthy rotor case. This phenomenon takes place in a continuously alternating or "swing-like" pendulous manner. In other words, the rotor's magnetic field orientation oscillates around the original direction in the healthy motor case, while the original direction rotates at synchronous speed

There is an additional factor influencing this angular displacement of the magnetic field orientation, namely that associated with the elevated degree of flux crowding and saturation level within the rotor iron laminations in the region surrounding the broken bars [7]. This aspect though has not been discussed here, will be revisited later on in this paper in relation to the experimental results. As one can see in this simple example, this continuously ongoing angular displacement of the magnetic field orientation will be a function of the number of broken bars and their position within the rotor geometry. In Section III, a method to detect the rotor magnetic field space vector orientation is presented using the space-vector theory in electric machines [8]

\section{SECTION III Space-Vector Formulation for Rotor Magnetic Field Orientation}

The space vectors of the stator current, voltage, or total flux linkage are defined as the sum of space vectors of individual phases as follows [8]:

$$
\vec{\Omega}_{s}(t)=\frac{2}{3}\left\{\Omega_{s a}(t)+\alpha \Omega_{s b}(t)+\alpha^{2} \Omega_{s c}(t)\right\}
$$

(6)

where the symbol $\Omega$ stands for the current $i$, voltage $v$, or total flux linkage $\lambda$ in the stator windings, and $\alpha=e^{j 2 \pi / 3}$ is a space operator. Meanwhile, the space-vector relationship between the stator voltage, and the total flux linkage in the stationary frame of reference is expressed as follows [8]: 


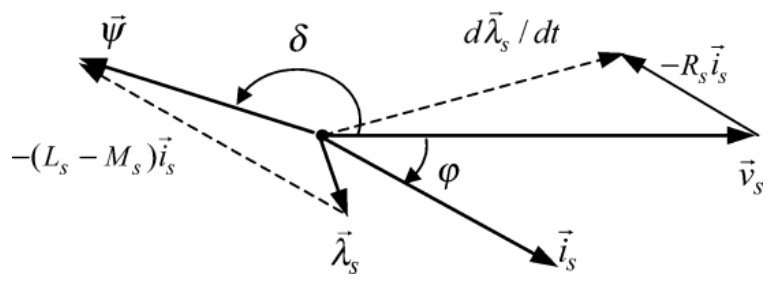

Fig. 4 Steady-state space vectors of stator voltage $\vec{v}_{s}$, stator current $\vec{i}_{s}$, stator flux linkage $\vec{\lambda}_{s}$, and the information signal $\vec{\psi}$

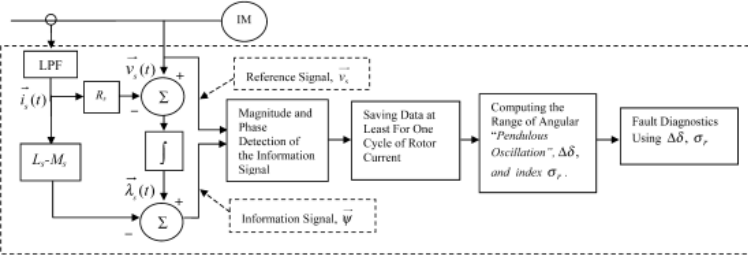

Fig. 5 Functional diagram of the rotor magnetic field orientation observer for broken-bar and shorted turn detection

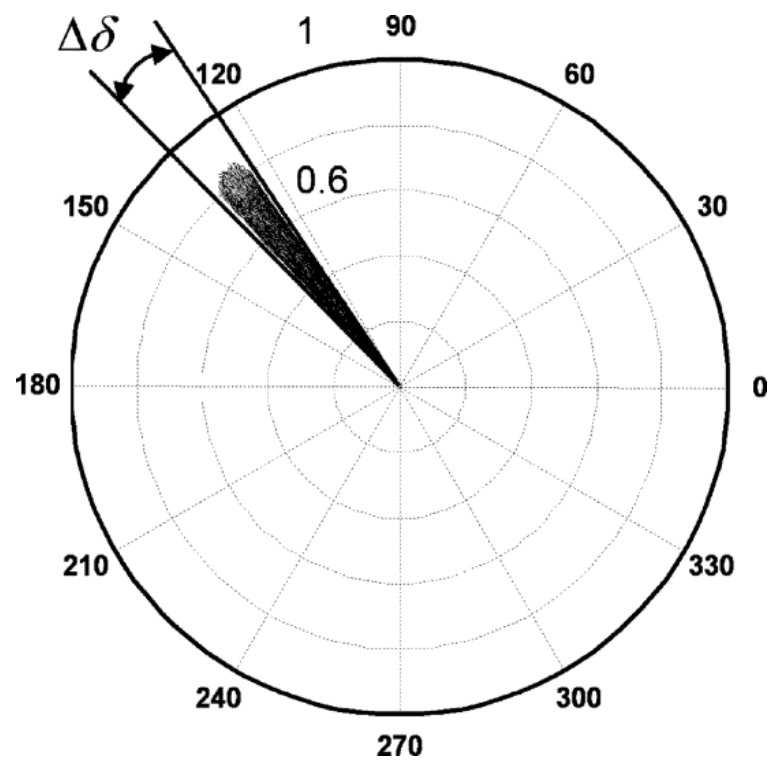

Fig. 6 Simulation-based results of the absolute value of $\psi_{\text {real }}$ in webers versus angle $\delta=\left(\angle \vec{\psi}(t)-\angle \vec{v}_{S}(t)\right)$ in degrees for a three-broken-bar fault

$$
\vec{v}_{s}(t)=R_{s} \vec{i}_{s}(t)+\frac{d \vec{\lambda}_{s}}{d t}
$$

(7)

and in induction machines, the space vector of the stator flux $\vec{\lambda}_{s}$ can be expressed in terms of the stator and rotor current space vectors as follows [8]:

$$
\vec{\lambda}_{s}(t)=\left(L_{s}-M_{s}\right) \vec{i}_{s}(t)+\frac{3}{2} M_{s r} \vec{i}_{r}^{\prime}(t)
$$


where $\vec{i}_{s}(t), \vec{v}_{s}(t)$, and $\vec{\lambda}_{s}(t)$ are as defined above in $(6)$. In these equations, $L_{s}$ and $M_{s}$ are the selfand mutual inductances of the stator phases, $L_{s}-M_{S}$ is the total three-phase stator inductance, and $M_{s r}$ is the peak value of the stator phase to rotor loop mutual inductance. In general, the aforementioned inductances might be functions of time when the effects of magnetic saturation and/or slotted air gaps are considered in a case study. Meanwhile, $\overrightarrow{i_{r}^{\prime}}(t)$, which is the space vector of the rotor currents referred to the stator side and expressed in the stationary reference frame, can be written in terms of the rotor loop currents for a cage with $N_{b}$ bars as follows:

$$
\overrightarrow{i_{r}^{\prime}}(t)=\left(\frac{2}{3} \sum_{k=1}^{N_{b}} e^{j\left(\frac{2 \pi(k-1)}{N_{b}}\right)} i_{L k}(t)\right) e^{-j \theta_{r}}
$$

Physically, the space vector of the rotor currents determines the instantaneous magnitude and instantaneous spatial orientation (displacement) of the peak of the rotor MMF distribution produced by the $N_{b}$ rotor loops. Therefore, in order to detect the rotor magnetic field orientation, one way is to detect (observe) the orientation of the second term on the right side of (8). Namely, this complex signal, $\vec{\psi}(t)=(3 / 2) M_{s r} \overrightarrow{i_{r}^{\prime}}(t)$, is graphically depicted in the phase-space vector diagram of Fig. 4 , through the use of $(7)$ and $(8)$. This observer signal $\vec{\psi}(t)$, the so-called information signal in Fig. 5 , can thus be expressed from $(8)$ as follows:

$$
\vec{\psi}(t)=\vec{\lambda}_{s}(t)-\left(L_{s}-M_{s}\right) \vec{i}_{s}(t)
$$

Now, we have established the background to present an observer for a rotor magnetic field spacevector orientation. This observer is mainly formed using (6), (7), and $(10)$ as outlined in the functional block diagram of this method depicted in Fig. 5. Accordingly, the stator winding parameters of the induction machine, $R_{S}, L_{s}$, and $M_{S}$ are used as the observer parameters (see Fig. 5). In reality, the machine parameters will slightly change due to the variations of temperature, voltage, and frequency of the operating conditions. Moreover, machine parameters vary due to the faults in the rotor bars and/or stator windings. However, in this work the parameters of this observer are set based on the healthy case values at the rated conditions of an induction machine. This results in an invariable (fixed) scale or reference from which to distinguish a healthy case from a broken-bar case, as well as help detect the number of broken bars for a given faulty condition as will be seen in the experimental results below

As explained above, in the case of a fault, the information signal oscillates around the healthy case orientation. If the induction motor is energized by a voltage source with balanced sinusoidal waveforms, obviously the stator voltage space vector $\vec{v}_{s}(t)$ will not have any pendulous oscillations. 
Hence, the pendulous oscillation of the information signal, $\vec{\psi}(t)$, is caused only by the space vector of the stator current

In the faulty case the rotor imperfections such as broken bars are the main contributor the modulations that take place in the space vector of stator current, which lead to the above mentioned pendulous oscillations in $\vec{\psi}(t)$. However, these oscillations can still exist, though to a considerably lesser extent, in the healthy motor case under test conditions. This is due mainly to imperfections (lack of perfect balance) in the location of the conductors in stator slots in a randomly wound motor such as the 2-hp test case presented below. This is in addition to the discrete and finite number of rotor bars, as well as lamination imperfections that disturb the magnetic symmetry around the $360^{\circ}$ structure of both the stator and rotor cores

\section{SECTION IV Fault Diagnostic Indexes and Analysis of Simulation Results}

A 230-V 60-Hz two-pole 2-hp squirrel-cage three-phase induction motor with 108 turns in each stator phase, 36 rotor bars, and 24 stator slots was simulated entirely in the $A B C$ frame of reference including the possibility of inter-turn shorts in stator windings and/or rotor broken-bar faults, using an approach of similar nature to the work of reference [9]. This machine was also tested under healthy and broken bar cases as will be given in the experimental results section of this paper. From knowledge of the $A, B$, and $C$ time-domain voltage and current waveforms, the information (observer) signal, $\vec{\psi}(t)$, was calculated according to $(10)$. This signal, $\vec{\psi}(t)$, which reveals the rotor magnetic field space-vector orientation, can be represented as follows:

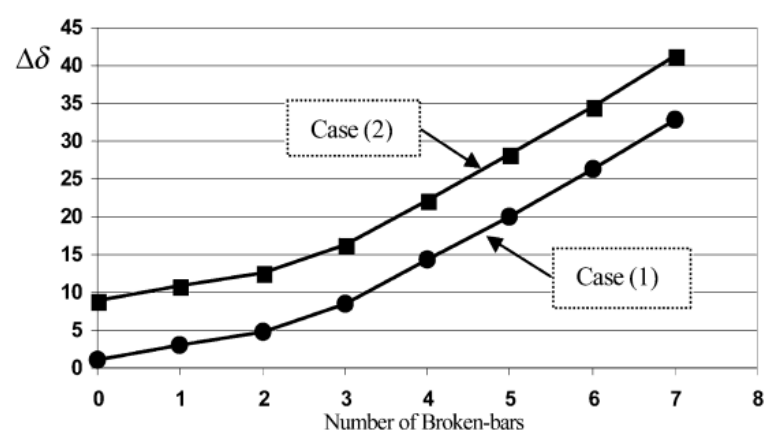

Fig. 7 Rotor field space-vector swing angle $\Delta \delta$ versus number of broken bars

$$
\vec{\psi}(t)=\psi_{\text {real }}+j \psi_{\text {imag }}
$$

In Fig. 6, the absolute value of $\psi_{\text {real }}$, that is $\left|\psi_{\text {real }}\right|$ in webers, with an orientation equal to the phase angle, $\delta=\left(\angle \vec{\psi}(t)-\angle \vec{v}_{s}(t)\right)$ in degree, is plotted in a polar coordinate diagram for the fault case of three broken bars, while the stator windings are in a healthy condition. The phase "swing" angle, $\Delta \delta$, of the observation signal was also computed (simulated) for the case of one through seven broken bars. It was found, as expected based on our earlier simple eight-bar example that $\Delta \delta$ is progressively increasing with the increase in the number of broken bars. The resulting plot, case (1), of the swing angle $\Delta \delta$ versus the number of broken bars obtained from the simulation is shown in Fig. 7. 


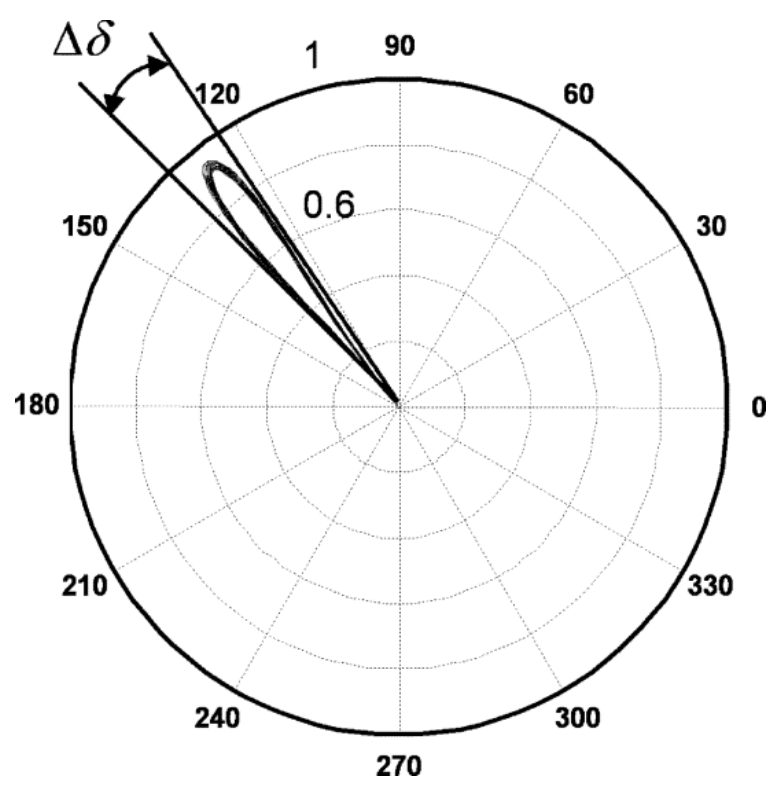

Fig. 8 Simulation-based results of the absolute value of $\psi_{\text {real }}$ in webers versus angle $\delta=\left(\angle \vec{\psi}(t)-\angle \vec{v}_{s}(t)\right)$ in degrees for an inter-turn stator winding short-circuit fault

Here, there is a definite relationship or proportionality correlation between the number of broken bars and the magnitude of the angle $\Delta \delta$. In these authors' judgment, this correlation can be exploited for diagnostics purposes

In order to ascertain whether this present approach can distinguish a stator shorted turn fault from a rotor broken-bar type of fault, the case of an inter-turn shorted stator winding was also modeled and simulated using a technique which was presented in an earlier paper by these authors [10]. In this shorted turns simulation, a concept of state-space modeling of the dynamics of induction motors using "line-to-line flux linkages," in conjunction with shorted turn formulations similar to those detailed in [11]were used. Accordingly, based on the results of this simulation, the absolute value of $\psi_{\text {real }}$ versus the phase angle $\delta$ is plotted in polar format in Fig. 8 for a case of one shorted turn in one of the these phases of the stator winding, while the rotor is assumed to be in a healthy condition. In these figures, $\Delta \delta$ demonstrates the range of the pendulous oscillations in the phase angle $\delta$. Moreover, in Fig. 7, the range of the pendulous oscillations is shown starting with a healthy rotor condition with progression up to the case with seven broken bars for a healthy stator winding condition, labeled case (1). The reasons of the small but nonzero value of pendulous oscillation angle, $\Delta \delta$, for the healthy motor case were discussed above at the end of Section III. Meanwhile, the simulation was repeated with an additional fault of one turn short in one of the phases in the stator winding, case (2). As one can see in Fig. 7, the observed swing angle for three broken bars with a healthy stator, case (1), is almost the same as the healthy rotor condition with a one turn-to-turn short within phase $A$ of the stator winding. 


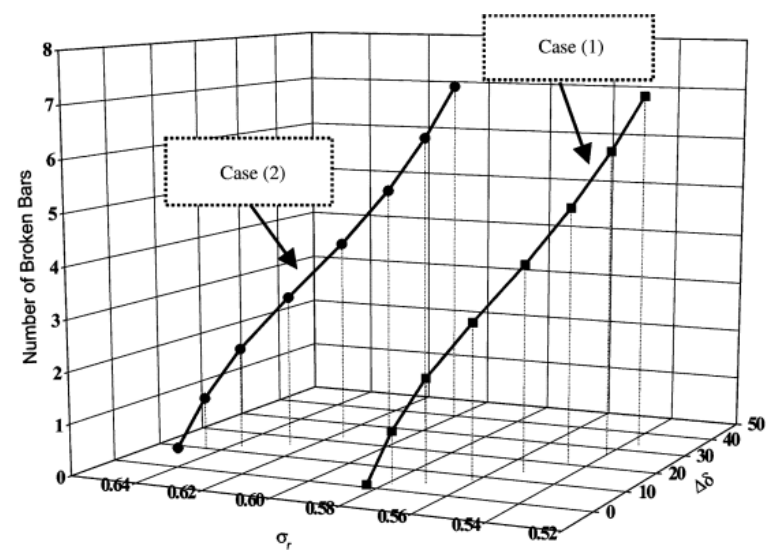

Fig. 9 Complementary index $\sigma_{r}$ to distinguish broken-bar faults from broken-bar plus shorted-turn faults, and pendulous oscillation $\Delta \delta$

Therefore, only observing the swing angle is not sufficient for distinguishing between a stator type of fault and a rotor broken-bar type of fault. Hence, a complementary variance index, $\sigma_{r}$, was introduced to aid in distinguishing a broken-bar fault from a fault of an inter-turn short in a stator winding. This variance index, $\sigma_{r}$, is defined here as follows:

$$
\sigma_{r}^{2}=\frac{\sum_{k=1}^{N} \psi_{\text {real }}^{2}\left(t_{k}\right)}{N-1}
$$

where $N$ is the number of data samplings covering at least one rotor current cycle. Use of this index $\sigma_{r}$ in conjunction with the phase "swing" angle $\Delta \delta$ yields plots such as shown in the threedimensional plot of Fig. 9. These plots demonstrate how stator-winding inter-turn faults occurring simultaneously with broken-bar faults can be distinguished from pure broken-bar faults through the swing angle $\Delta \delta$ in conjunction with the distinct values of the new variance index $\sigma_{r}$. See the set of faults in case (1) involving only broken bars, and the set of faults in case (2) involving a simultaneous occurrence of rotor broken-bar and stator shorted turn faults in Fig. 9

\section{SECTION V Experimental Results and Their Analysis}
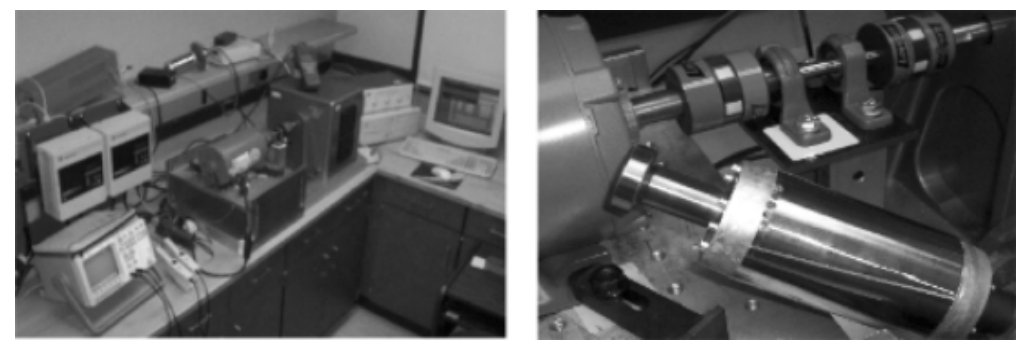

Fig. 10 (a) Laboratory setup for the experimental verification and (b) a rotor with five broken bars 


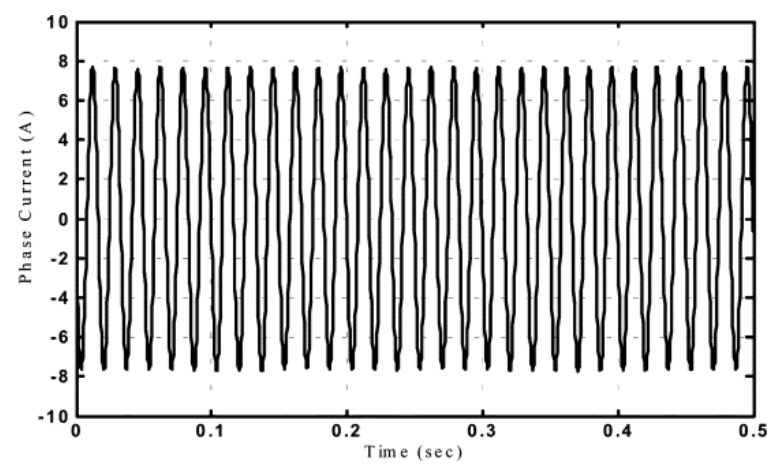

Fig. 11 Stator line current profile over a one-rotor slip cycle from testing of a healthy rotor case

The 2-hp three-phase squirrel-cage induction motor, which was considered for simulation purposes in Section IV, was also tested in the laboratory under healthy operation, as well as under one through five broken-bar faults (at 1.8-hp load); see the test setup in Fig. 10. This new angular rotor field orientation swing angle method was applied to the experimental data of observed stator current and voltage waveforms to obtain the swing angle $\Delta \delta$ of the pendulous oscillation of the rotor field orientation. As sample examples, the stator current waveforms for a rotor frequency cycle $s f$, are given in Figs. 11 and 12 for the healthy and five-broken-bar faults, respectively. Meanwhile, the FFT spectral analysis was also applied to the same experimentally obtained stator currents used in the rotor field swing angle analysis. Here, Figs. 13, 15, 17, and 19 show the swing angle $\Delta \delta$ under healthy operation as well as one-, three-, and five-broken-bar faults, respectively. In these figures, the absolute value of $\psi_{\text {real }}$ in webers, with an orientation equal to the phase angle $\delta=\left(\angle \vec{\psi}(t)-\angle \vec{v}_{s}(t)\right)$ in degree, are plotted in a polar coordinate diagram. The swing angle $\Delta \delta$ under healthy operation as well as one through five broken-bar faults are given with the corresponding number of broken bars in Table I. The values of $\Delta \delta$ versus the number of broken bars are also plotted in Fig. 21 . As can be clearly observed from the data in Table I and the plot of Fig. 21, the swing angle $\Delta \delta$ bears a definite correlation to the number of broken bars, whereby one can observe that the larger the value of $\Delta \delta$, the larger the number of broken bars

Meanwhile, Figs. 14, 16, 18, and 20 show the normalized FFT spectrum of the stator current for the same set of experimental data starting with the healthy (no broken bars) case, as well as the faulty cases of one, three, and five broken bars, respectively. In these figures, the sideband components are shown at frequencies $f_{b}=(1 \pm 2 s) f$, here, $s=0.0333$

The following queries have to be answered by a fault diagnostics system.

1. Is the rotor cage healthy or unhealthy?

2. If there are broken bars, is their number on the increase? 


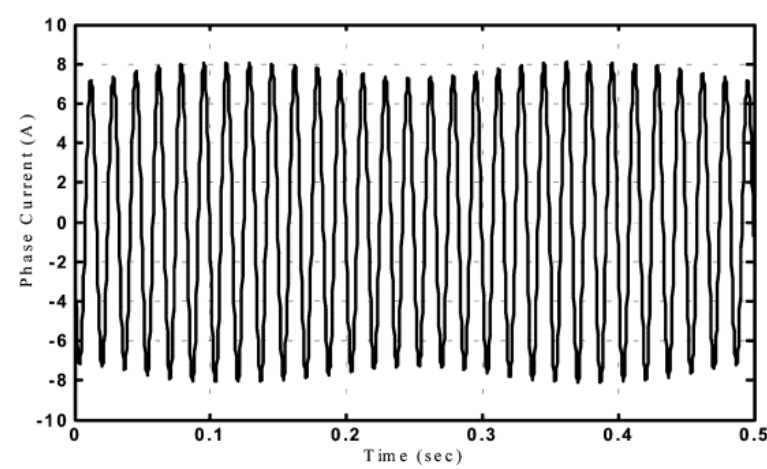

Fig. 12 Stator line current profile over a one-rotor slip cycle from testing of a five-broken-bar case

The first query can be answered by using the swing angle $\Delta \delta$ as well as the sideband component. It may be argued that for a fault case constituting only one broken bar the side band components can answer the first query with a higher resolution than the swing angle, $\Delta \delta$. However, the sideband component magnitudes are function of the load torque and inertia [12], while the swing angle $\Delta \delta$ can be observed even at no-load condition. Moreover, in order to observe the side band component, normally a slip (speed) measurement is necessary, while in order to observe the swing angle $\Delta \delta$, only the synchronous speed is needed, which is readily available from knowledge of the space-vector angle of the stator voltage, $\angle \vec{v}_{S}(t)$. The speed (slip) measurement can be achieved either directly using a tachometer or estimated indirectly from the stator voltages and currents. The direct measurement adds a device to an online diagnostic system, therefore it reduces the reliability and increases the cost of the diagnostic system. Meanwhile, the indirect measurement provides a suitable estimation for control applications, where a tolerance is inherent in such a procedure, and such a tolerance value may not be suited for diagnostics purposes. In order to judge whether a broken bar fault is becoming a more sever fault or not, the second query must be answered. This query is answered in detail in Section VI.

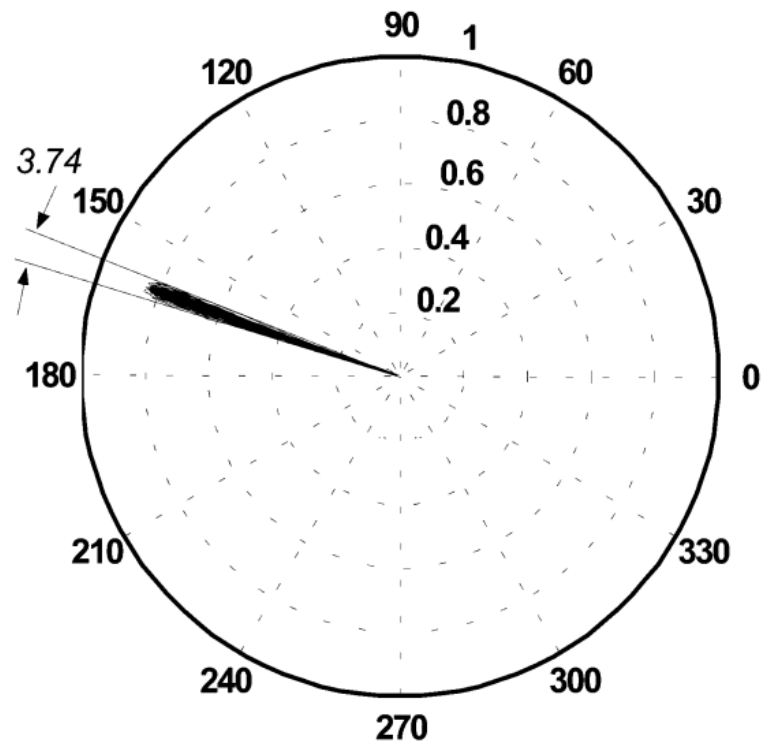

Fig. 13 Experimentally based results of the absolute value of $\psi_{\text {real }}$ in webers versus angle $\delta=(\angle \vec{\psi}(t)-$ $\left.\angle \vec{v}_{S}(t)\right)$ in degrees for a healthy motor case 
TABLE I Swing Angle $\Delta \delta$ and Sideband Maqnitudes for the Healthy and one Through Five Broken-Bar Faults

\begin{tabular}{|l|l|l|}
\hline Rotor situation & $\Delta \delta$ (Degrees) & Side Band Magnitude $(\mathrm{db})\left(\right.$ at $\left.f_{b}=(1-2 s) f\right)$ \\
\hline Healthy Rotor & 3.7423 & -58.2 \\
\hline One Broken-Bar & 4.4907 & -44.4 \\
\hline Two Broken-Bars & 5.7250 & -36.8 \\
\hline Three Broken-Bars & 8.1056 & -32.6 \\
\hline Four Broken-Bars & 10.6307 & -29.5 \\
\hline Five Broken-Bars & 16.2255 & -25.2 \\
\hline
\end{tabular}

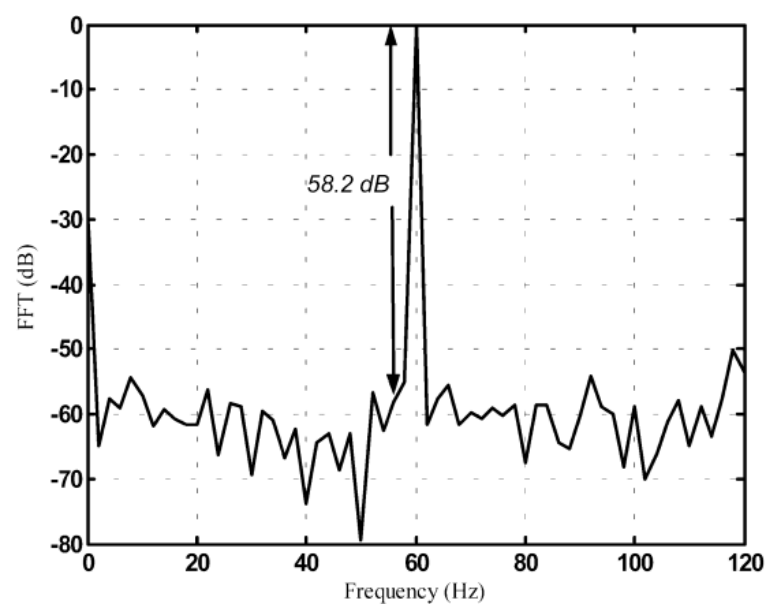

Fig. 14 Normalized FFT spectrum of the test stator line current for a healthy motor case

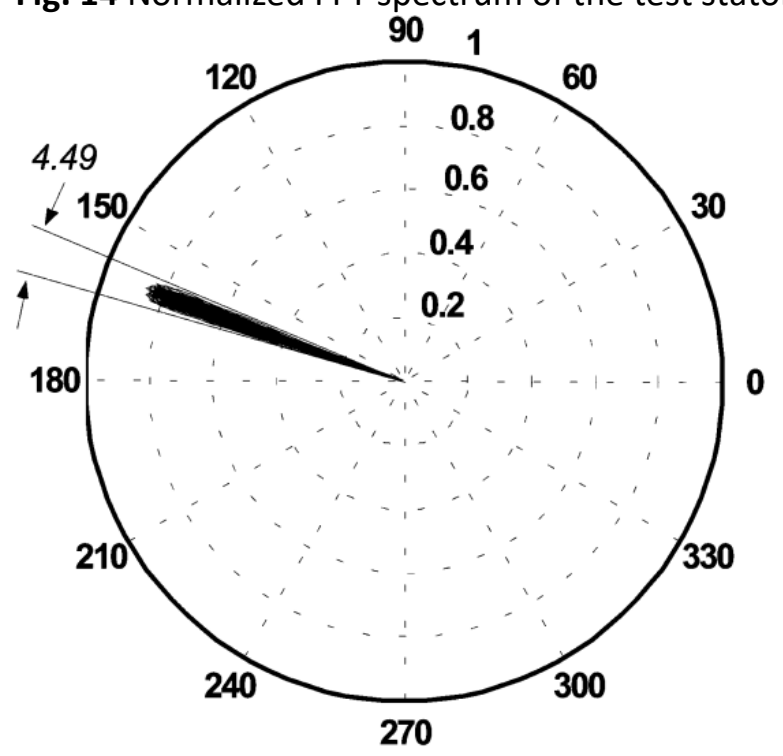

Fig. 15 Experimentally based results of the absolute value of $\psi_{\text {real }}$ in webers versus angle $\delta=(\angle \vec{\psi}(t)-$ $\left.\angle \vec{v}_{S}(t)\right)$ in degrees for a one-broken-bar fault 


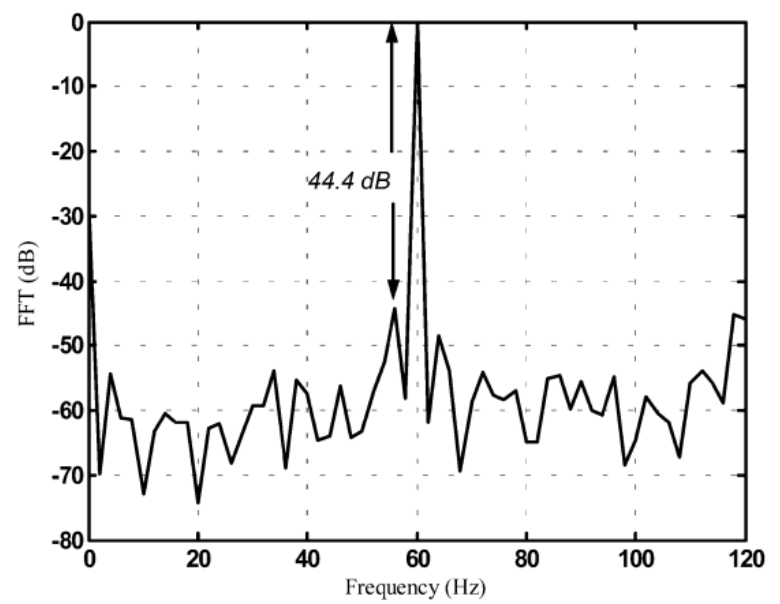

Fig. 16 Normalized FFT spectrum of the test stator line current for a one-broken-bar fault

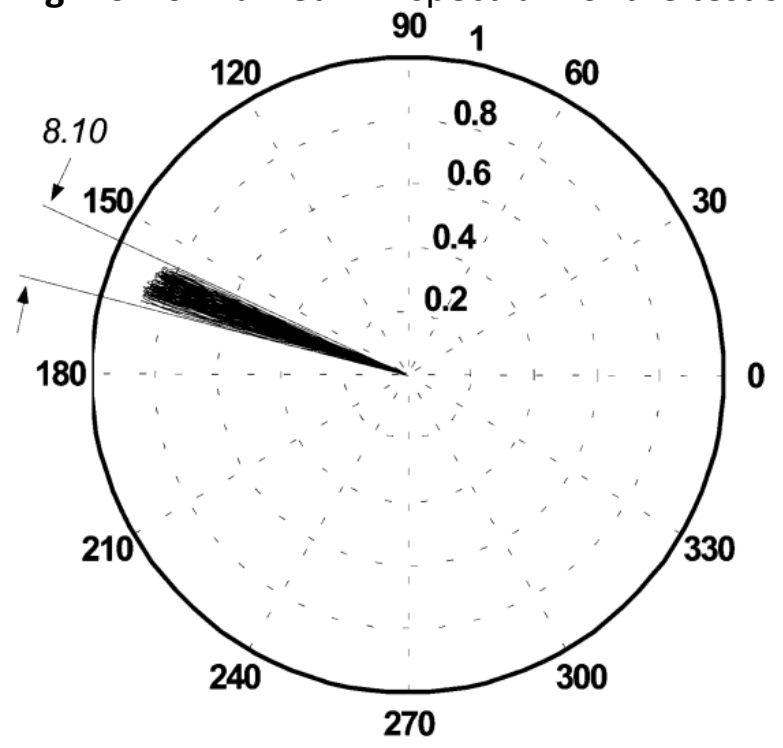

Fig. 17 Experimentally based results of the absolute value of $\psi_{\text {real }}$ in webers versus angle $\delta=$ $\left(\angle \vec{\psi}(t)-\angle \vec{v}_{S}(t)\right)$ in degrees for a three-broken-bar fault

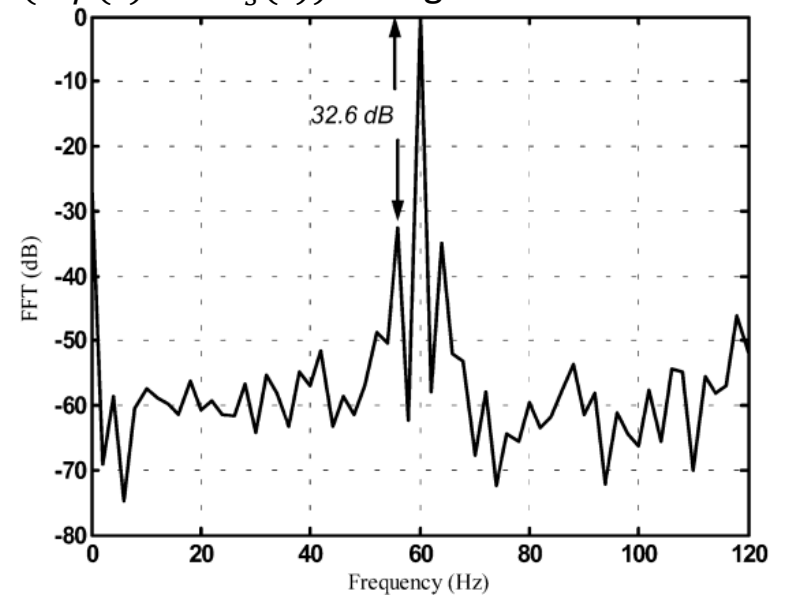

Fig. 18 Normalized FFT spectrum of the test stator line current for a three-broken-bar fault 


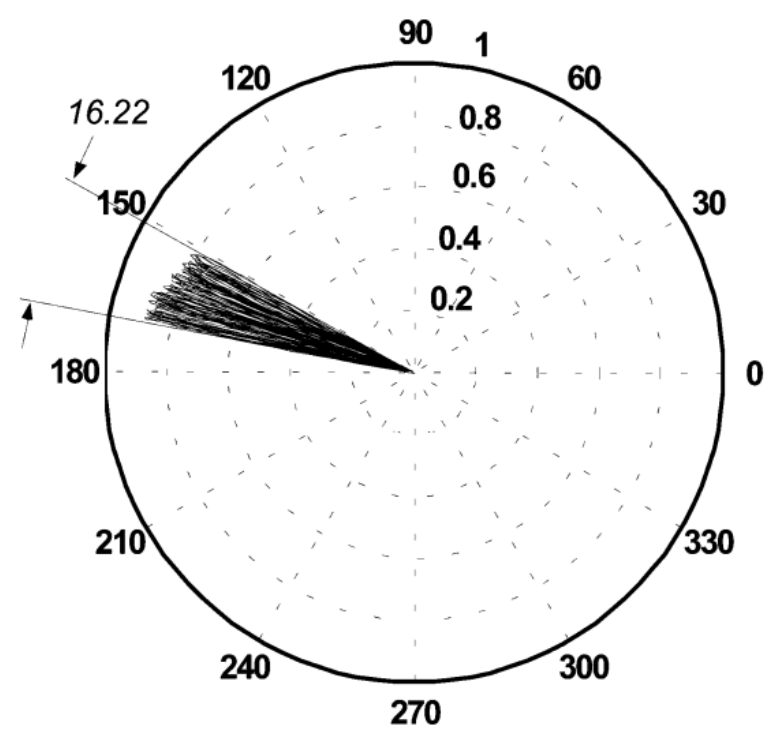

Fig. 19 Experimentally based results of the absolute value of $\psi_{\text {real }}$ in webers versus angle $\delta=$ $\left(\angle \vec{\psi}(t)-\angle \vec{v}_{s}(t)\right)$ in degrees for a five-broken-bar fault

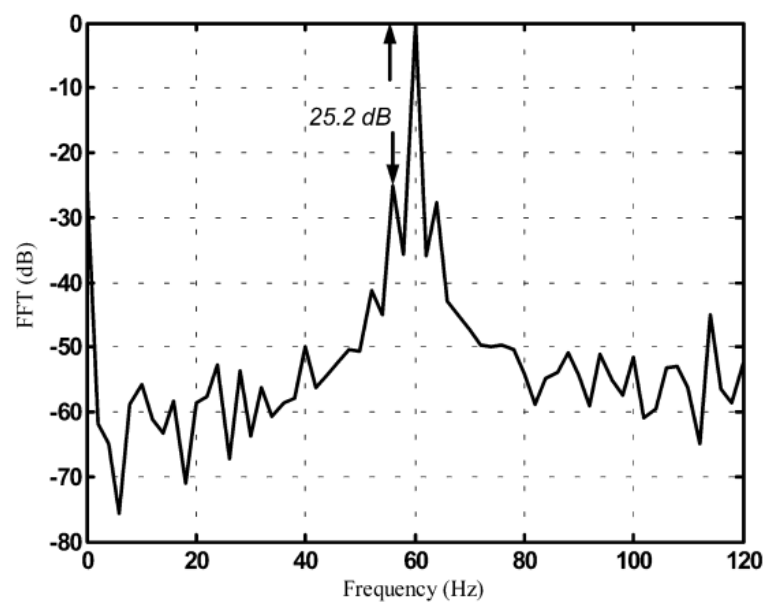

Fig. 20 Normalized FFT spectrum of the test stator line current for a five-broken-bar fault

SECTION VI Discussion of the Sideband Components Versus the Swing Angle The second query, in Section V, must be answered to judge whether a broken-bar fault is becoming a more severe fault or not. Now, the question is: "Which one of these indexes, the swing angle $\Delta \delta \delta^{\circ}$ the FFT sideband component, can provide a stronger criterion."

Upon close examination of Figs. 21and 22, it can be realized that:

1. in the " swing angle" case, Fig. 21, we have

$$
\frac{d(\Delta \delta)}{d n_{b}}>0 \text { and } \frac{d^{2}(\Delta \delta)}{d n_{b}{ }^{2}}>0
$$

2. while in the "FFT sideband" case, Fig. 22, we have 


$$
\frac{d(d b)}{d n_{b}}>0 \text { and } \frac{d^{2}(d b)}{d n_{b}{ }^{2}} \neq 0( \pm)
$$

where $n_{b}$ indicates the number of adjacent broken bars. Expressions (13)and (14) show that the swing angle $\Delta \delta$ can clearly indicate whether the number of broken bars is increasing because of the positive value of the second derivative $d^{2}(\Delta \delta) / d n_{b}{ }^{2}$, while that is not the case for the FFT sideband method because the second derivative $d^{2}(d b) / d n_{b}{ }^{2}$ may either be positive or negative. Accordingly, the swing angle $\Delta \delta$ and its first derivative $d(\Delta \delta) / d n_{b}$ are both increasing monotonic functions. This can be attributed to the direct physical relationship between $\Delta \delta$ and the extent of distortion in the rotor MMF due to the increase in the number of broken bars. It should be further pointed out that an increase in the number of broken bars is accompanied by an increase in the severity of the degree of magnetic saturation in the rotor lamination region surrounding the broken bars as shown in earlier work [7], thus leading indirectly to further increase in the swing angle, $\Delta \delta$. On the contrary, for the stator spectrum sideband component, the first derivative $d(d b) / d n_{b}$ is always positive, however, it is not an increasing monotonic function. This can be attributed to the weaker physical link between the degree of stator current waveform distortion resulting from broken bars, and any increase in the number of broken bars. Therefore, the swing angle $\Delta \delta$ provides a stronger criterion to judge whether a broken bar fault is becoming a more severe fault or not.

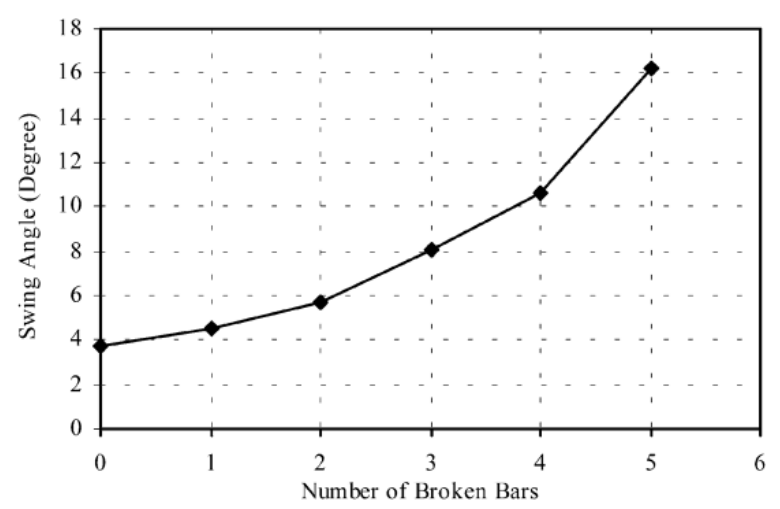

Fig. 21 Rotor field space-vector swing angle $\Delta \delta$ versus number of broken bars obtained from test results

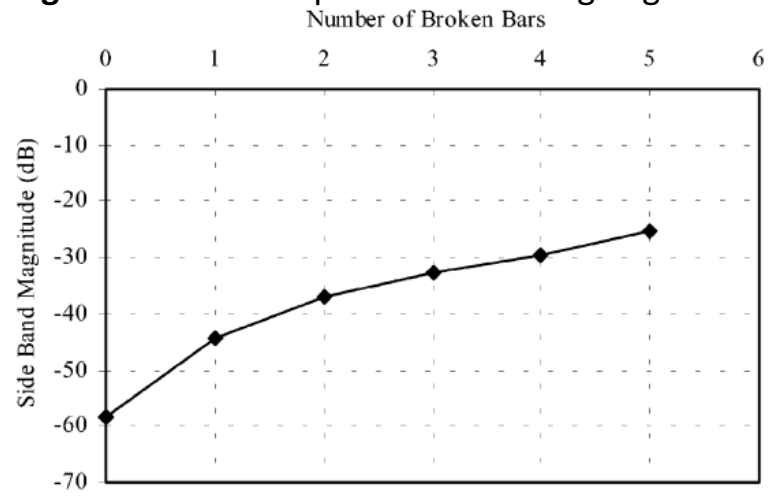

Fig. 22 Normalized line-current sideband magnitude at $f_{b}=(1-2 s) f$ versus number of broken bars 


\section{SECTION VII Conclusion}

A new technique based on the "swing-like" pendulous oscillation of the rotor magnetic field orientation has been developed and presented for rotor broken-bar fault detection. An associated variance index derived from the pendulous swing angle has been developed to distinguish a rotor broken-bar fault from an inter-turn stator-winding short circuit when such faults occur concurrently. Mainly, this paper has shown that as the number of broken bars increases, the range of the "swinglike" pendulous oscillation of the rotor magnetic field orientation will increase in a monotonically unambiguous manner. This phenomenon has been verified experimentally for broken-bar faults in a case-study squirrel-cage induction motor

\section{ACKNOWLEDGMENT}

The authors wish to express their deep appreciation for the support rendered by S. J. Dellinger, Dr. D. M. lonel, and R. P. Bartos of A. O. Smith Corporation in providing the test motor and spare rotor

\section{References}

1. P. Vas, Parameter Estimation Condition Monitoring and Diagnosis of Electrical Machines, New York:Oxford Univ. Press, 1993.

2. M. E. H. Benbouzid, "Bibliography on induction motors faults detection and diagnosis", IEEE Trans. Energy Conversion, vol. 14, pp. 1065-1074, Dec. 1999.

3. D. Kostic-Perovic, M. Arkan and P. Unsworth, "Induction motor fault detection by space vector angular fluctuation", Conf. Rec. IEEE-IAS Annu. Meeting, vol. 1, pp. 388-394, 2000.

4. M. Haji and H. A. Toliyat, "Pattern recognitionA technique for induction machines rotor broken bar detection", IEEE Trans. Energy Conversion, vol. 16, pp. 312-317, Dec. 2001.

5. R. M. Tallam, T. G. Habetler and R. G. Harley, "Stator winding turn-fault detection for closedloop induction motor drives", Conf. Rec. IEEE-IAS Annu. Meeting, vol. 3, pp. 1553-1557, 2002.

6. R. Povinelli, J. F. Bangura, N. A. O. Demerdash and R. H. Brown, "Diagnostics of bar and end-ring connector breakage faults in polyphase induction motors through a novel dual track of timeseries data mining and time-stepping coupled FE-state space modeling", IEEE Trans. Energy Conversion, vol. 17, pp. 39-46, Mar. 2002.

7. J. F. Bangura and N. A. O. Demerdash, "Diagnosis and characterization of effects of broken bars and connectors in squirrel-cage induction motors by a time-stepping coupled finite elementstate space modeling approach", IEEE Trans. Energy Conversion, vol. 14, pp. 1167-1176, Dec. 1999.

8. P. Vas, Electrical Machines and Drives; A Space-Vector Theory Application, New York:Oxford Univ. Press, 1992.

9. H. A. Toliyat and T. A. Lipo, "Transient analysis of cage induction machines under stator rotor bar and end ring faults", IEEE Trans. Energy Conversion, vol. 10, pp. 241-247, June 1995.

10. B. Mirafzal and N. A. O. Demerdash, "A nonlinear controller for current source inverter induction motor systems", Proc. IEEE Int. Electric Machines and Drives Conf. IEMDC 2003, vol. 3, pp. 1491-1497, 2003.

11. R. M. Tallam, T. G. Habetler and R. G. Harley, "Transient model for induction machines with stator winding turn faults", IEEE Trans. Ind. Applicat., vol. 38, pp. 632-637, May/June 2002. 
12. Bellini, F. Filippetti, G. Franceschini, C. Tassoni and J. Kliman, "Quantitative evaluation of induction motor broken bars by means of current signatures", IEEE Trans. Ind. Applicat., vol. 37, pp. 1248-1255, Sept./Oct. 2001. 